\title{
Response to "Rates of Inhibitor Development in Previously Untreated Patients with Severe Hemophilia A Treated with Plasma-Derived or Recombinant Factor VIII: No Proof of Difference or Proof of No Difference?"
}

\author{
Massimo Franchini, MD ${ }^{1}$ Carlo Mengoli, MD² \\ ${ }^{1}$ Department of Hematology and Transfusion Medicine, Carlo Poma \\ Hospital, Mantua, Italy \\ 2 University of Padua, Padua, Italy
}

Semin Thromb Hemost 2014;40:271-272.
Address for correspondence Massimo Franchini, MD, Department of Hematology and Transfusion Medicine, Carlo Poma Hospital, Mantua, Italy (e-mail: massimo.franchini@aopoma.it).
We thank Messori et al for their letter ${ }^{1}$ regarding our metaanalysis on the role of factor VIII (FVIII) product type in inhibitor development in previously untreated patients (PUPs) with severe hemophilia A, recently published in Seminars in Thrombosis $\mathcal{E}$ Hemostasis. ${ }^{2}$ We highly appreciate their technical reanalysis of our data, which gives us another opportunity to point out some aspects of our research.

Equivalence and noninferiority methods are practically ignored or not performed within a meta-analysis. Even in the most recent version of the Review Manager documentation of Cochrane Collaboration, this topic is not mentioned except for a passing reference. ${ }^{3}$ Indeed, this procedure to formulate the null and the alternative hypothesis is used by original studies, mostly performed for registration purposes, when the new drug is anticipated as not being superior to the established therapy. This should be flanked by other advantages ignored by tests examining the main issue of pharmacological potency, such as lower cost, easer administration, fewer collateral adverse effects, or other aspects of better convenience. In these circumstances, and because the proof of exact equality is impossible to produce, a prestated margin of admissible, negligible inferiority is defined (delta). Delta tends to be smaller than the clinically relevant margin proposed for superiority trials, so the sample size of noninferiority trials tends to be larger. $^{4}$

In our meta-analysis, the original included studies were almost exclusively clinical, phase IV-observational reports not conforming to rules for noninferiority. Moreover, they did not declare a delta. We also did not declare a delta. The main task of a meta-analysis is to monitor the relevant literature to judge whether the available published evidence supports a decision to change established practices and guidelines. Accepting the delta (4\%) suggested by Messori et al, our study would have been inconclusive/indeterminate. However, the number of patients required to enroll in a single "megatrial," to obtain a modest power $=0.5$ for a one-sided $p=0.05$, would have been much higher than that included in our analysis, and would have comprised 5,188 if the two arms were of equal size (sample calculated using the R nBinomial function, according to Anderson). ${ }^{5}$ Our previously published meta-analysis was able to detect the absence of a significant difference in the incidence of inhibitors using plasma-derived or recombinant FVIII. ${ }^{2}$ The direct comparison between plasma-derived and recombinant FVIII products was not significant by logistic mixed model analysis, as reported in the following table of coefficients ( - Table $\mathbf{1}$ ).

Certainly, this does not provided a proof of an equivalence of the two types of FVIII preparation. Moreover, we affirmed neither that the incidence of inhibitors was the same or "equivalent" in the two groups of treated patients nor that equivalence or noninferiority was demonstrated.

Finally, we stress from a qualitative point of view the results of our research. Indeed, this meta-analysis was an update of a previous systematic review, ${ }^{6}$ but the addition of a significant number of patients (more than 600) did not modify the results of a lack of statistically significant difference in inhibitor rate between PUPs treated with plasmaderived and recombinant FVIII products. In particular, our conclusions were in line with those of a very large, published online February 5, 2014
Issue Theme Quality in Hemostasis and Thrombosis, Part III; Guest Editors, Emmanuel J. Favaloro, PhD, FFSc (RCPA), Giuseppe Lippi, MD, and Mario Plebani, MD.
Copyright @ 2014 by Thieme Medical Publishers, Inc., 333 Seventh Avenue, New York, NY 10001, USA. Tel: +1(212) 584-4662.
DOI http://dx.doi.org/ $10.1055 / \mathrm{s}-0034-1367472$. ISSN 0094-6176. 
Table 1 Logistic regression showing the effect of FVIII product type on inhibitor development

\begin{tabular}{|l|l|l|l|l|l|}
\hline Inhibitors & Coefficients & Standard error & $z$ & $p$ & $95 \% \mathrm{Cl}$ \\
\hline FVIII type $^{\mathrm{a}}$ & 0.2694 & 0.2490 & 1.08 & 0.279 & -0.2185 to 0.7574 \\
\hline Intercept & -1.2684 & 0.1994 & -6.36 & 0.000 & -1.6592 to -0.8776 \\
\hline
\end{tabular}

Abbreviation: $\mathrm{Cl}$, confidence interval.

Note: Multilevel, mixed effects logistic regression, where the dependent variable was the proportion (cumulative incidence) of patients with inhibitors. Observations $=1,340$. Group variable: studies, number of $=$ groups $=25$, log likelihood $=-780.25248$.

${ }^{\mathrm{a}} \mathrm{FVIII}$ type $=1$ : recombinant; FVIII type $=0$ : plasma.

observational trial, the RODIN study, ${ }^{7}$ which alone accounted for approximately half of the total number of patients included in our meta-analysis. Furthermore, the recent update of the European Haemophilia Safety Surveillance registry confirmed the similar rate of inhibitor development for recombinant and plasma-derived products. ${ }^{8}$

In conclusion, while we are aware that a more definite evidence on this still controversial issue will come only from the ongoing prospective randomized SIPPET study, ${ }^{9}$ we strongly believe that, on the basis of the results of our meta-analysis, the suggested difference in the inhibitor rate between plasma-derived and recombinant FVIII products should not be advocated for the choice of the type of FVIII product to administer to hemophilia A patients. Vice versa, in our opinion the therapeutic choice should be driven rather by the technological improvements of the different products aimed at the minimization of the risk of blood-borne virus transmission and at the amelioration of patients' compliance and quality of life through innovative modalities of FVIII concentrate administration. In other words, there is actually insufficient evidence from the literature to modify the recommendation from the Guidelines of Italian Association of Hemophilia Centers (AICE) regarding the use of recombinant FVIII products as first-line therapy in PUPs. ${ }^{10}$

\section{References}

1 Messori A, Fadda V, Maratea D, Trippoli S. Rates of inhibitor development in PUPs with severe haemophilia A treated with plasma-derived or recombinant Factor VIII: no proof of difference or proof of no difference? [Letter to the Editor] Semin Thromb Hemost 2014;40(2):269-270

2 Franchini M, Coppola A, Rocino A, et al; Italian Association of Hemophilia Centers (AICE) Working Group. Systematic review of the role of FVIII concentrates in inhibitor development in previously untreated patients with severe hemophilia a: a 2013 update. Semin Thromb Hemost 2013;39(7):752-766

3 Manager R. (RevMan) [Computer program]. Version 5.2. Copenhagen: The Nordic Cochrane Centre, The Cochrane Collaboration; 2012. Available at: http://www.cochrane.org/. Accessed: October 31, 2013

4 Piaggio G, Elbourne DR, Altman DG, Pocock SJ, Evans SJ; CONSORT Group. Reporting of noninferiority and equivalence randomized trials: an extension of the CONSORT statement. JAMA 2006; 295(10):1152-1160

5 Anderson K. Package "gsDesign". Available at: http://cran.rproject.org/web/packages/gsDesign/gsDesign.pdf. Accessed: October 31,2013

6 Franchini M, Tagliaferri A, Mengoli C, Cruciani M. Cumulative inhibitor incidence in previously untreated patients with severe hemophilia A treated with plasma-derived versus recombinant factor VIII concentrates: a critical systematic review. Crit Rev Oncol Hematol 2012;81(1):82-93

7 Gouw SC, van der Bom JG, Ljung R, et al; PedNet and RODIN Study Group. Factor VIII products and inhibitor development in severe hemophilia A. N Engl J Med 2013;368(3):231-239

8 Makris M, Calizzani G, Hay C, et al. European monitoring of inhibitor development in hemophilia A and B. J Thromb Haemost 2013;11(Suppl 2):133 (abstract)

9 Mannucci PM, Gringeri A, Peyvandi F, Santagostino E. Factor VIII products and inhibitor development: the SIPPET study (survey of inhibitors in plasma-product exposed toddlers). Haemophilia 2007;13(Suppl 5):65-68

10 Santagostino E, Mannucci PM, Bianchi Bonomi A. Guidelines on replacement therapy for haemophilia and inherited coagulation disorders in Italy. Haemophilia 2000;6(1):1-10 